\title{
Semantic priming by irrelevant speech
}

\author{
Jan P. Röer ${ }^{1}$ - Ulrike Körner ${ }^{1} \cdot$ Axel Buchner ${ }^{1} \cdot$ Raoul Bell $^{1}$
}

Published online: 31 October 2016

(C) Psychonomic Society, Inc. 2016

\begin{abstract}
To-be-ignored, task-irrelevant speech disrupts serial recall performance relative to a quiet control condition. In most studies, the content of the auditory distractors had no effect on their disruptive potential, one's own name being one of the few exceptions. There are two possible explanations of this pattern: (1) Semantic features of the irrelevant speech are usually not processed, except for highly relevant auditory distractors, or (2) semantic processing of the irrelevant speech always occurs, but usually does not affect serial recall performance. To test these explanations, we presented to-be-ignored auditory distractor words drawn from different categories while participants memorized visual targets for serial recall. Afterwards, participants were invited to what they believed to be an unrelated norming study, in which they were required to spontaneously produce words from the categories from which the auditory distractor words were drawn. Previously ignored words were produced with a higher probability than words from a parallel, nonpresented set, demonstrating that features of to-be-ignored, task-irrelevant speech that do not interfere with immediate serial recall performance are nevertheless processed semantically and may have substantial effects on subsequent behavior.
\end{abstract}

Keywords Working memory $\cdot$ Attentional capture $\cdot$ Semantic priming $\cdot$ Short-term memory

Electronic supplementary material The online version of this article (doi:10.3758/s13423-016-1186-3) contains supplementary material, which is available to authorized users.

Jan P. Röer

jan.roeer@hhu.de

1 Department of Experimental Psychology, Heinrich Heine University Düsseldorf, 40225 Düsseldorf, Germany
The processing of auditory information is unavoidable in a way that the processing of visual information is not. Unlike eyes, ears cannot be closed to block off unwanted input. This openness may have the advantage that important auditory stimuli (e.g., one's own name, the crying of a baby, a fire alarm) can be detected even in an unattended channel. However, it comes at the disadvantage of enhanced disruptability by task-irrelevant information. A classic example for such a disruption is the irrelevant speech effect on serial recall. In a typical experiment, participants recall a visually presented list of to-be-remembered items in their correct order. When task-irrelevant speech is played while the list is memorized, serial recall performance is reduced relative to a quiet control condition (Parmentier \& Beaman, 2015; Röer, Bell, \& Buchner 2014b, 2015; Schlittmeier, Weißgerber, Kerber, Fastl, \& Hellbrück 2012). Usually the research focus lies on the detrimental aspects of the processing of irrelevant speech on cognitive performance (i.e., how much of the relevant information is lost). In the present study, we concentrated on the fate of the ignored information instead (i.e., how much of the irrelevant information is available).

The question of whether to-be-ignored speech is processed semantically has been addressed by researchers for more than half a century (Broadbent, 1958; Deutsch \& Deutsch, 1963). In many early experiments, a dichotic listening task was used, in which participants are required to attend to a message presented to one ear while ignoring a second message presented to the other ear. Based on a literature review, Bentin, Kutas, and Hillyard (1995) suggested that the content of to-beignored speech is always processed to some extent, but whether or not it affects overt behavior depends on this processing surpassing a critical level. In line with such a view, recent evidence suggests that semantic processing of to-be-ignored speech in dichotic listening depends critically upon acoustic parameters such as the intensity of the irrelevant information (Aydelott, Jamaluddin, \& Nixon Pearce 2015). It remains 
unclear, however, whether these findings from unimodal distraction paradigms simply can be generalized to cross-modal distraction paradigms. In irrelevant speech experiments, all auditory input must be ignored and it has been argued recently that in such a situation the auditory information can be efficiently blocked off at subcortical processing levels (Guerreiro, Murphy, \& Van Gerven 2010). Indeed, in most studies the content of the irrelevant speech had little or no effect on its potential to disrupt serial recall performance. It is a common finding, for example, that serial recall performance is unaffected by whether the irrelevant speech is played forward or backward (Jones, Miles, \& Page 1990; Röer, Bell, \& Buchner 2014a). The magnitude of the irrelevant speech effect is also independent of the degree of semantic similarity between items and distractors (Buchner, Irmen, \& Erdfelder 1996; Marsh, Hughes, \& Jones 2009). On the rare occasion that the semantic features of irrelevant speech did exacerbate interference - such as the own name compared to the name of a yoked-control partner (Röer, Bell, \& Buchner 2013) and negatively valent words compared to neutral words (Buchner, Rothermund, Wentura, \& Mehl 2004) - highly self-relevant or emotional stimuli were presented, which are known for their attention-grabbing potential.

With regard to the question of whether irrelevant speech is processed semantically, or not, there are at least two possible explanations of these results. One possibility is that the magnitude of interference may indicate to which degree semantic features of the irrelevant speech are processed in working memory. This would imply that for the most part the to-beignored material is not processed at a semantic level, except when attention-grabbing stimuli are presented such as one's own name. Indeed, one's own name appears to be a special case. Even during sleep the auditory presentation of one's own name triggers a differential brain response as compared to other names (Perrin, Garcia-Larrea, Mauguiere, \& Bastuji 1999). This led Perrin et al. to speculate that one's own name can be detected without elaborated semantic processing, because it has immediate access to long-term memory due to its self-related emotional content and excessive repetition along life. Larger disruption of serial recall by one's own name compared to the name of a yoked-control partner (Röer et al., 2013) may just be another example of this principle. An alternative explanation is that the distractor features that interfere with serial recall are not indicative of the level to which the auditory information is processed. It is possible that semantic processing of irrelevant speech always occurs but that this processing usually does not cause interference with nonsemantic primary tasks, such as serial recall.

The question of whether irrelevant speech is processed semantically has important implications for working memory models such as the interference-by-process account (Marsh et al., 2009) and the embedded-processes model (Cowan, 1995). According to the interference-by-process account
(Marsh, Hughes, \& Jones 2008; Marsh et al., 2009), the irrelevant speech effect on serial recall is due to a conflict of relevant and irrelevant order information resulting from the obligatory processing of acoustic distractor features. The magnitude of disruption is solely determined by changes in the amplitude and frequency spectrum, semantic distractor features are only assumed to interfere with tasks that rely heavily on semantic processing. Two interpretations of this account are possible (Marsh, Röer, Bell, \& Buchner, 2014). First, it could be postulated that the content of the distractors is not processed unless the primary task stimulates semantic processing, which cannot be confined to the relevant material, and may "spill over" to the distractor material. Alternatively, the semantic content of the distractors may always be processed, but this processing may not affect task performance unless the primary task relies on semantic processing. As yet, it is unclear which of the two interpretations is correct.

According to the embedded-processes model (Cowan, 1995) irrelevant speech disrupts serial recall because it elicits an orienting response that draws attentional resources away from the maintenance of the target item list. Two types of stimuli are assumed to particularly capture attention: (1) unexpected stimuli, and (2) highly significant stimuli. The strength of the embedded-processes model is that it can readily explain larger irrelevant speech effects for highly significant stimuli such as one's own name or negatively valent words. It is unclear, however, whether all stimuli are processed semantically in order to determine their significance, or not. In fact, Cowan (1995) states that "all incoming stimulation makes contact with long-term memory and activates some of the relevant features, but it is not yet certain if this includes semantic features" (p. 141). It therefore could be that semantic processing is limited to stimuli that capture a critical level of attention from the outset due to their nonsemantic features. For instance, the acoustic pattern of one's own name should be extremely familiar, which may suffice to capture that critical level of attention. Although the embeddedprocesses model is, in principle, compatible with semantic processing of distractor features, it does not directly predict that semantic processing occurs in cross-modal paradigms in which the distractors can be easily filtered out based on their physical features.

Thus, it is an open empirical question whether to-beignored, irrelevant speech is processed semantically, or not. The present study was designed to close this empirical gap with a direct assessment of semantic activation. To determine whether irrelevant speech is processed semantically, we presented auditory distractor words which (unbeknownst to the participants) were drawn from different semantic categories (e.g., musical instrument, four-legged animal, type of fruit). In a subsequent and seemingly unrelated "norming study," we asked our participants to produce spontaneously exemplars from these categories. A 
higher probability of producing previously ignored words would be evidence of the aftereffects of prior semantic processing, that is, of semantic priming.

\section{Experiment 1}

\section{Method \\ Participants}

Ninety Heinrich Heine University students (59 women) were paid for participating or received course credit. Their ages ranged from 18 to 37 years $(M=24.2, S D=4.7)$. All participants were fluent German speakers and reported normal hearing and normal or corrected-to-normal vision.

\section{Materials}

For each trial, eight to-be-remembered digits were sampled randomly without replacement from the set $\{1,2, \ldots, 9\}$. The digits were presented in black at a rate of 1 digit per second ( $800 \mathrm{~ms}$ on, $200 \mathrm{~ms}$ off) in 72-point Monaco font on a white background in the centre of the computer screen.

Each irrelevant speech sequence consisted of eight distractor words that were drawn from a single semantic category taken from the Mannhaupt (1983) norms. There were eight distractor trials in each of which a different semantic category was used. For each category, two different sets of words were created with the same mean production frequency. To avoid ceiling effects, we did not include the eight most frequently produced exemplars. The complete set of category-exemplars with production frequencies is listed in the supplemental material.

Auditory distractors were recorded using Apple's text-tospeech software that is part of Mac OS X 10.11. They were spoken by the female computer voice, "Anna," and were digitally recorded at $16 \mathrm{bit}, 44.1 \mathrm{kHz}$. The irrelevant speech sequences lasted 8 seconds with a rate of approximately 1 distractor per second. All sounds were presented binaurally at approximately $60 \mathrm{~dB}(\mathrm{~A})$.

\section{Procedure}

Participants wore headphones with high-insulation hearing protection covers, which were plugged directly into an Apple iMac computer. Standard written instructions informed the participants that any sound would be task-irrelevant and should be ignored.

In the first part of the experiment, participants completed 24 serial recall trials. The training phase consisted of eight quiet trials to familiarize participants with the task. The experimental phase consisted of eight trials in each of the two sound conditions (quiet, irrelevant speech), which were presented in random order. Participants were randomly assigned to one of the two distractor set groups. One group ignored distractors from Set 1; the other ignored distractors from Set 2. Irrelevant speech was only played during item presentation. Immediately after each trial, participants recalled the digits in the order of their presentation using the keyboard's number pad. Digits at a particular serial position could be omitted by pressing a "don't know" button. There was no possibility to correct a prior entry. Feedback was given after each trial. The first part of the study took approximately $14 \mathrm{~min}$ to complete.

After participants had left their cubicle, we asked them whether they wanted to take part in what they believed to be an unrelated norming study. To cover up that this was actually the second part of the experiment, participants filled out a separate form of consent and were asked once again for their personal data. Responses also were collected on a different medium (paper). Participants were required to produce exemplars spontaneously from the same categories from which the auditory distractors were drawn. Written instructions informed the participants that they should answer with the first category-exemplars that came to mind. For each category, there was a separate sheet, which had eight blank boxes on it. Participants were instructed to fill out each of these boxes, one after another (i.e., the first category-exemplar should be written in the first box, the second exemplar in the second box, and so on). Once eight category-exemplars had been produced, participants turned over the paper and proceeded with the next category. The order of categories was counterbalanced across participants. The second part of the study took approximately $10 \mathrm{~min}$ to complete.

The experimenter was instructed to write down if a participant reported suspicions that the serial recall task and the category-exemplar production test were part of the same experiment.

\section{Design}

A within-subject design was used with distractor set (Set 1, Set 2) as the independent variable and number of category-exemplars produced as the dependent variable. A higher production frequency for "old" words (i.e., previously ignored category-exemplars) compared with the production frequency for "new" exemplars (i.e., category-exemplars from the other set) would be evidence for semantic priming by irrelevant speech. Thus, the critical test is whether old category-exemplars are produced with a higher probability than new categoryexemplars, which is a one-tailed test problem. Given a total sample size of $N=90$ and $\alpha=\beta=0.05$, a semantic priming effect of size $d_{z}=0.35$ could be detected (Faul, Erdfelder, Lang, \& Buchner 2007). 


\section{Results}

Four participants reported noticing that the serial recall task and the category-exemplar production test were part of the same experiment. This had no effect on the statistical results, which is why we decided to include the data from these participants in our analyses.

Serial recall task Responses in the serial recall task were only scored correct when the digits were reproduced at the exact serial position at which they had been presented. The typical irrelevant speech effect was observed. Participants made more errors in serial recall when auditory distractors were played as compared to the quiet condition, $F(1,88)=83.28, p<0.001$, $\eta_{\mathrm{p}}{ }^{2}=0.49$. There was no effect of distractor set, $F(1,88)=$ $0.01, p=0.945, \eta_{\mathrm{p}}^{2}<0.01$, and, most importantly, no interaction of sound condition and distractor set, $F(1,88)=0.85, p$ $=0.360, \eta_{\mathrm{p}}{ }^{2}=0.01$. Serial recall performance is listed in Table 1.

Category-exemplar production test As expected, dominant category-exemplars according to the Mannhaupt (1983) norms (i.e., the eight most frequently produced category-exemplars) were produced with a higher probability than category-exemplars from Set $1, t(89)=32.90, p<0.001, \eta_{\mathrm{p}}{ }^{2}$ $=0.92$, and from Set 2, $t(89)=28.51, p<0.001, \eta_{\mathrm{p}}{ }^{2}=0.90$. There was no difference in the overall production frequencies for category-exemplars from Set 1 and Set 2, $t(89)=-0.60, p$ $=0.549, \eta_{\mathrm{p}}{ }^{2}<0.01$.

The critical question was whether the production frequency would be higher for category-exemplars from the previously ignored distractor set than for category-exemplars from the control set. That is, we examined whether participants who had previously ignored distractors from Set 1 would produce more category-exemplars from Set 1 than from Set 2 and vice versa. Across both groups, participants produced categoryexemplars from the previously ignored set with a higher probability than category-exemplars from the control set, $t(89)=$ $3.33, p=0.001, \eta_{\mathrm{p}}{ }^{2}=0.11$. Separate analyses for both distractor sets confirmed this pattern of results (Fig. 1). Participants who had ignored category-exemplars from Set 1 produced more exemplars from Set 1 than from Set 2, $t(44)=$

Table 1 Serial recall performance as a function of distractor set (Set 1, Set 2) and sound condition (quiet, irrelevant speech) in Experiment 1

\begin{tabular}{llllll}
\hline & \multicolumn{2}{l}{ Set 1} & & & \multicolumn{2}{l}{ Set 2 } & \\
\cline { 2 - 3 } \cline { 5 - 6 } & $\mathrm{M}$ & SEM & & $\mathrm{M}$ & SEM \\
\hline Quiet & 0.75 & 0.02 & & 0.77 & 0.02 \\
Irrelevant Speech & 0.63 & 0.03 & & 0.62 & 0.03 \\
\hline
\end{tabular}

2.17, $p=0.035, \eta_{\mathrm{p}}{ }^{2}=0.10$, and those who had ignored category-exemplars from Set 2 produced more exemplars from Set 2 than from Set 1, $t(44)=2.51, p=0.016, \eta_{\mathrm{p}}{ }^{2}=0.13$.

The priming score was $P_{\mathrm{c}}=0.023$. The score was calculated as the proportion of ignored exemplars produced minus the base rate of producing these exemplars without prior exposure (the base rate for Set 1 was obtained from the production frequencies of participants who had ignored categoryexemplars from distractor Set 2 , and vice versa). This is equivalent to a $15 \%$ higher probability to produce a word that was previously presented as an auditory distractor compared with the base value of producing the same word if it was not presented before.

Although the mean production frequency was higher for category-exemplars from the previously ignored sets than for those from the control sets, there was no difference in the mean output position when category-exemplars from the ignored sets were compared with category-exemplars from the control sets, $t(89)=0.55, p=0.586, \eta_{\mathrm{p}}^{2}<0.01$.

\section{Experiment 2}

Although we used the same procedure and materials as in studies in which the content of the auditory distractors had no effect on serial recall performance (Marsh et al., 2008; 2009), there may be a remote possibility that the semantic content of the irrelevant speech had captured attention for unknown reasons. To make sure that this was not the case, we ran a second experiment in which we directly compared the disruptive effect of the irrelevant speech sequences used in Experiment 1 with a control condition in which the semantic processing of the distractor material was not possible.

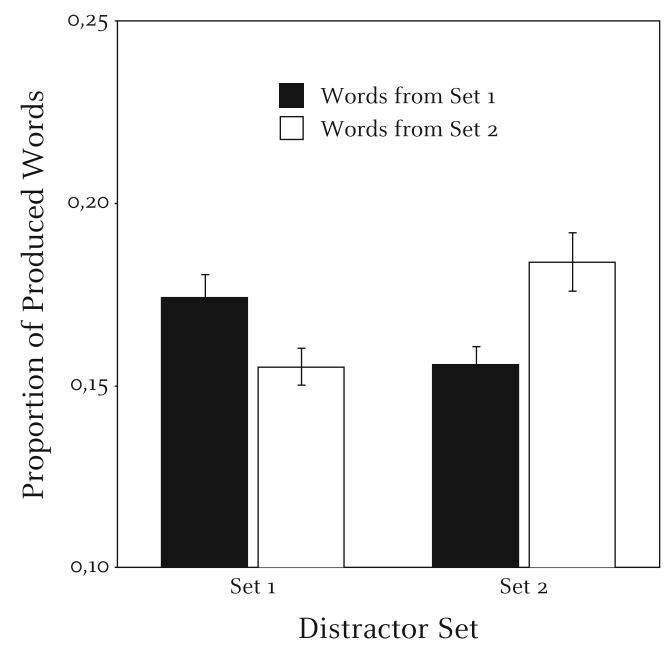

Fig. 1 Proportion of words from Set 1 and Set 2 that were written down in the category-exemplar production test as a function of distractor set (Set 1, Set 2). The error bars depict the standard errors of the means 


\section{Method}

\section{Participants}

Ninety Heinrich Heine University students (71 women) were paid for participating or received course credit. Their ages ranged from 18 to 38 years $(M=23.6, S D=4.6)$ All participants were fluent German speakers and reported normal hearing and normal or corrected-to-normal vision.

\section{Materials, procedure, and design}

The serial recall task was the same as that of Experiment 1 . There were three sound conditions: (1) a quiet control condition, (2) a forward irrelevant speech condition in which the stimuli from Set 1 used in Experiment 1 were played forward, and (3) a backward irrelevant speech condition in which the stimuli from Set 1 used in Experiment 1 were played backward. The experiment took approximately $20 \mathrm{~min}$ to complete.

\section{Results}

The typical effect of irrelevant speech on serial recall performance was observed, that is, participants made more errors in the two distractor conditions relative to the quiet control condition, $F(1,89)=122.16, p<0.001, \eta_{\mathrm{p}}{ }^{2}=0.58$. Most importantly, the disruptive effect of speech played forward was not different from that of speech played backward, $F(1,89)=0.03$, $p=0.597, \eta_{\mathrm{p}}{ }^{2}<0.01$. Serial recall performance is listed in Table 2.

\section{Discussion}

In Experiment 1, previously ignored words were produced with a higher probability in the category-exemplar production test than words that were not presented in the experiment, which is evidence of semantic priming by irrelevant speech. From the outset, it was unclear whether there would be semantic priming because in most studies the content of the

Table 2 Serial recall performance as a function of sound condition (quiet, forward irrelevant speech, backward irrelevant speech) in Experiment 2

\begin{tabular}{lll}
\hline & M & SEM \\
\hline Quiet & 0.74 & 0.02 \\
Forward Irrelevant Speech & 0.61 & 0.02 \\
Backward Irrelevant Speech & 0.61 & 0.01 \\
\hline
\end{tabular}

auditory distractors had little or no effect on their potential to disrupt serial recall performance (Buchner et al., 1996; Marsh et al., 2009). Experiment 2 once again confirms that irrelevant speech played forward is no more disruptive than irrelevant speech played backward, which cannot be processed semantically. Thus, a lack of interference does not seem to imply that the content of the distractor words is not processed. On the contrary, Experiment 1 demonstrates that features of the irrelevant speech that have been shown not to interfere with ongoing performance may nonetheless have a significant effect on overt behavior in a subsequent task.

According to the interference-by-process account (Marsh et al., 2008; 2009), the content of irrelevant speech is only assumed to interfere with tasks that rely heavily on semantic processing. Thus far, it was unclear whether semantic distractor features are simply not processed unless semantic processing of the relevant material "spills over" to the processing of the distractor material or whether they are always processed, but do not interfere with nonsemantic primary tasks such as serial recall. The present results clarify that semantic features of irrelevant speech are indeed processed even in the absence of an effect on serial recall performance. Thus, it seems possible to conclude that auditory distractors are always processed semantically to some extent but that it only causes interference if the nature of the primary task necessitates semantic processing as well (Marsh et al., 2008; 2009). Note, however, that the semantic content of the auditory distractors may capture attention away from the primary task if the sound is of particular significance for the individual (Röer et al., 2013).

While the embedded-processes model (Cowan, 1995) readily predicts attentional capture by significant stimuli, it does not yield a clear prediction of whether semantic processing should be limited to these type of stimuli or whether all stimuli must be processed semantically in order to determine their significance. The present results indicate that irrelevant speech is always processed semantically to some extent; however, the semantic processing does not, in itself, capture and hold attention unless the stimulus is highly self-relevant (Röer et al., 2013) or emotionally salient (Buchner et al., 2004). Against the backdrop of this model, the semantic processing of the distractors could be conceptualized as an adaptive feature of the cognitive system that enables the detection of potentially important (e.g., self-relevant) stimuli in an unattended channel.

In sum, the findings presented provide new insights into the fate of ignored information in memory. Although the content of irrelevant speech often is of no significance to the disruption of working memory performance, it still may have a substantial effect on subsequent behavior, suggesting that semantic priming effects are a more sensitive measure of the degree to which irrelevant auditory information is processed than errors in serial recall. This also has practical implications, in 
that not only acoustical features must be considered when aiming to minimize the influence of auditory distractors on cognitive performance, but also their intelligibility. A lack of interference with ongoing activity does not imply that the content of irrelevant speech has no effect on a subsequent task.

Acknowledgments The research was supported by Grant RO 4972/1-1 from the Deutsche Forschungsgemeinschaft (German Research Foundation).

\section{References}

Aydelott, J., Jamaluddin, Z., \& Nixon Pearce, S. (2015). Semantic processing of unattended speech in dichotic listening. The Journal of the Acoustical Society of America, 138, 964-975.

Bentin, S., Kutas, M., \& Hillyard, S. A. (1995). Semantic processing and memory for attended and unattended words in dichotic listening: Behavioral and electrophysiological evidence. Journal of Experimental Psychology: Human Perception and Performance, 21, 54-67.

Broadbent, D. E. (1958). Perception and communication. New York: Pergamon.

Buchner, A., Irmen, L., \& Erdfelder, E. (1996). On the irrelevance of semantic information for the "Irrelevant Speech" effect. The Quarterly Journal of Experimental Psychology A: Human Experimental Psychology, 49A, 765-779.

Buchner, A., Rothermund, K., Wentura, D., \& Mehl, B. (2004). Valence of distractor words increases the effects of irrelevant speech on serial recall. Memory \& Cognition, 32, 722-731.

Cowan, N. (1995). Attention and memory: An integrated framework. Oxford University Press.: Oxford University Press.

Deutsch, J. A., \& Deutsch, D. (1963). Attention - some theoretical considerations. Psychological Review, 70, 80-90.

Faul, F., Erdfelder, E., Lang, A.-G., \& Buchner, A. (2007). G*Power 3: A flexible statistical power analysis program for the social, behavioral, and biomedical sciences. Behavior Research Methods, 39, 175-191.
Guerreiro, M. J., Murphy, D. R., \& Van Gerven, P. W. (2010). The role of sensory modality in age-related distraction: A critical review and a renewed view. Psychological Bulletin and Review, 136, 975-1022.

Jones, D. M., Miles, C., \& Page, J. (1990). Disruption of proofreading by irrelevant speech: Effects of attention, arousal or memory? Applied Cognitive Psychology, 4, 89-108.

Mannhaupt, H.-R. (1983). Produktionsnormen für verbale Reaktionen zu 40 geläufigen Kategorien. Sprache und Kognition, 4, 264-278.

Marsh, J. E., Hughes, R. W., \& Jones, D. M. (2008). Auditory distraction in semantic memory: A process-based approach. Journal of Memory and Language, 58, 682-700.

Marsh, J. E., Hughes, R. W., \& Jones, D. M. (2009). Interference by process, not content, determines semantic auditory distraction. Cognition, 110, 23-38.

Marsh, J. E., Röer, J. P., Bell, R., \& Buchner, A. (2014). Predictability and distraction: Does the neural model represent post-categorical features? PsyCH Journal, 3, 58-71.

Parmentier, F. B. R., \& Beaman, C. P. (2015). Contrasting effects of changing rhythm and content on auditory distraction in immediate memory. Canadian Journal of Experimental Psychology, 69, 28-38.

Perrin, F., Garcia-Larrea, L., Mauguiere, F., \& Bastuji, H. (1999). A differential brain response to the subject's own name persists during sleep. Clinical Neurophysiology, 110, 2153-2164.

Röer, J. P., Bell, R., \& Buchner, A. (2013). Self-relevance increases the irrelevant speech effect: Attentional disruption by one's own name. Journal of Cognitive Psychology, 25, 925-931.

Röer, J. P., Bell, R., \& Buchner, A. (2014a). Evidence for habituation of the irrelevant sound effect on serial recall. Memory \& Cognition, 42, 609-621.

Röer, J. P., Bell, R., \& Buchner, A. (2014b). What determines auditory distraction? On the roles of local auditory changes and expectation violations. PLoS One, 9, e84166.

Röer, J. P., Bell, R., \& Buchner, A. (2015). Specific foreknowledge reduces auditory distraction by irrelevant speech. Journal of Experimental Psychology: Human Perception and Performance, 41, 692-702.

Schlittmeier, S. J., Weißgerber, T., Kerber, S., Fastl, H., \& Hellbrück, J. (2012). Algorithmic modeling of the irrelevant sound effect (ISE) by the hearing sensation fluctuation strength. Attention, Perception \& Psychophysics, 74, 194-203. 